\title{
Biotechnology and animal health
}

\section{By Christopher R. Stokes, Department of Veterinary Medicine, University of Bristol, Langford House, Langford, Bristol BS18 7DU}

The potential areas of impact for biotechnology on the control of infectious diseases and improved animal health are manifold. They include the identification of susceptible stock, tests for the rapid and early diagnosis of disease, an understanding of the mechanisms of pathogenesis and the development of safe and effective vaccines or prophylactics. The principal methodologies which will enable these targets to be approached are those of monoclonal-antibody production and recombinant-DNA technology.

The benefits of selecting stock on the basis of resistance to specific infectious agents would appear at present to be restricted to those areas of the world where the importance of a single disease predominates over all others (e.g. trypanosomiasis). Whilst the possibility of selecting on an antigen non-specific basis is likely to be of more general relevance, at present the development of vaccination strategies that can effectively control infectious agents is likely to be of greatest significance for improved animal health. To this end monoclonal antibodies can be used in the development of diagnostic sera and reagents, and the characterization of virulence and adhesion determinants. Once identified such determinants can be isolated in a pure form from crude mixtures with monoclonal immunosorbents. Infectious diseases may be controlled by the prophylactic use of monoclonal antibodies, but this direct effect will be of limited use in animal production, by comparison with their potential use as 'educators' of the immune system in anti-idiotype vaccines.

Recombinant-DNA technology can provide a range of potential immunogens including attenuated or non-pathogenic strains and subunit vaccines. Since the majority of infectious agents are met at mucosal surfaces, the problem that remains is how these vaccines may be delivered so as to provide effective presentation and so stimulate a protective response.

\section{Mucosal defence}

The level of challenge to, and the degree of sterility maintained differs markedly between the various mucosal surfaces; from the gastrointestinal and upper respiratory tracts to the lower respiratory tract, reproductive tract and mammary gland which at least in health remain sterile. They are presented with a vast array of different antigens ranging from potential pathogens to relatively harmless dietary components. Together with the skin they provide the interface between the host and its environment. However, unlike the skin there are other physiological roles (e.g. gaseous exchange, absorption of nutrients, etc.) which restrict their ability to keep antigens out. Since the mucosal surfaces provide such a primary point of contact it is at these sites that the products of biotechnology might be expected to provide the most relevant and beneficial effects. For convenience the major part of the present review will be restricted to antigens presented to the gut.

The immune system of the gastrointestinal tract is presented with two types of antigens, those capable of causing infection and those, such as dietary antigens, which in themselves impose no direct threat to the animal's health. The type of response stimulated is critical, for whilst failure to respond to potential pathogens may be fatal, an 
over-reaction to dietary antigens may lead to damaging hypersensitivity responses (for review, see Newby \& Stokes, 1984a). The latter may in turn lead to altered handling of potential pathogens (Stokes et al. 1986). Therefore, although the most obvious area of impact of biotechnology will be directly on infectious agents, regulating responses to food antigens may also be of significance.

The response to food antigens. Three responses have been described that may protect an individual from damaging hypersensitivity reactions to dietary antigens. Immune exclusion mediated by locally produced IgA may reduce the absorption of intact proteins across the gut mucosae and in certain species it has been shown that any antigen that is absorbed may be eliminated via bile. These processes which restrict the access of antigenic material to the immune system are not absolute and the development of an acquired specific hyporesponsive state is probably of greater significance. Thus orally induced tolerance may actively down-regulate the response to dietary proteins. Before the induction of this 'protected state' all animals pass through a transient phase of hypersensitivity, during which time damaging allergic reactions can be demonstrated. In farm species it has been shown that following weaning, piglets are sensitive to antigens in the post-weaning diet and since these changes occur before the proliferation of Escherichia coli it has been postulated that response to food proteins may be an important predisposing factor in the aetiology of post-weaning diarrhoea (Miller et al. 1984). The importance of gut flora and bacterial lipopolysaccharide in regulating the response to dietary antigens has been clearly shown in rodents (Newby \& Stokes, 1984a), thus by the use of engineered strains of bacteria it may be possible to regulate the response to dietary proteins and so prevent the transient allergic responses that reduce the gut's ability to handle potential pathogens.

This brief description of the regulation of the response to food proteins attempts to serve two purposes. First, it may indicate a role for engineered strains in the controls of hypersensitivity reactions and the consequences thereof. Second, the type of response generated to food proteins differs markedly from that required to eliminate infectious agents. It is then perhaps not surprising, as will be discussed later, that dead mucosal microbial vaccines are generally not very effective.

The response to infection. The implications of biotechnology on animal health directly related to infections are numerous; it is therefore proposed to discuss them sequentially from diagnosis to vaccine development.

The conventional methods for the identification of the causal agent have in the past depended heavily on an ability to isolate and grow the organism in pure culture. The use of monoclonal antibodies should aid both the identification of infectious agents and their virulence factors. Unlike conventional anti-serums, which by virtue of the nature of the immune response are very heterogenous, monoclonal proteins have a unique specificity which allows such discrimination to be made. For example, by use of the enzyme-linked immunosorbent assay (ELISA) it is possible to detect subtle antigen differences between strains of an organism as well as to characterize the immune response of the infected animal.

Once having identified a particular virus, for example as being important in the pathogenesis of diarrhoea, how then might one proceed toward an effective vaccine? Consider for instance that virus $\mathrm{X}$ has been shown to be an important pathogen but is closely related to similar commensals. It can produce disease, and animals that recover from infection show solid protection. In neutralization tests it has been possible to show the specificity of $X$, but it is very difficult to grow! Further progress may then involve the development of a sensitive immunoassay for research and sero-epidemiology. Production of a monoclonal antibody (either conventionally from rodents or preferably from 
infected farm species) may allow isolation of a strain-specific antigen. Subsequently the antigen should be produced by cloning and expressing the gene in, for example $E$. coli, by recombinant-DNA technology. Such a procedure may form the basis for the development of a vaccine.

\section{Control of infection}

Without attempting to consider ways of eradicating an organism from the environment there are two types of approach to the prevention-control of infection within an animal. The first is essentially passive and involves little or no requirement of the host's immune system, whilst the second requires the stimulation of an active vigorous immune response.

Passive protection. Monoclonal antibodies have been used therapeutically to passively protect neonatal pigs and calves against challenge with a lethal dose of a $\mathrm{K} 99$ positive pathogenic strain of $E$. coli (Muscoplat, 1983). It has been pointed out that such an approach has a theoretical advantage over conventional vaccination of the dam, in that it does not require anticipation of the problem. Further, in this case where the disease is restricted to the first $24 \mathrm{~h}$ of life and the effects of the bacteria are contained within the intestine, it is directly treated by oral administration of the monoclonal antibody. A similar approach has been used to protect mice from intranasal infection with Respiratory Syncital Virus (Taylor et al. 1983), but generally it would appear that the therapeutic use of monoclonal antibodies will be of benefit in specific, defined individual areas, notably in human medicine, rather than on a herd basis in veterinary medicine. The rationale for this argument is the amount of antibody required, particularly if it is necessary that it is given orally. Monoclonal antibodies to infectious agents may, however, be of benefit in the 'education' of the immune system and this active process will be discussed later.

Another passive form of treatment involves the use of anti-microbials, and in this context biotechnology has already made a significant impact. Human leucocyte interferon has been isolated by monoclonal anti-immunosorbents (Secher \& Burke, 1980) and on a commercial level recombinant interferon and interleukin 2 are now generally available.

Active protection. Before considering the potential impact of biotechnology on the development of safe, effective vaccines, it is necessary to consider what is required of such products in order that they may stimulate a protective response. As has already been mentioned, the vast majority of infectious agents are met at a mucosal surface and therefore vaccines should be directed toward stimulating protection at these sites. To this end it is clear that by far the most effective method of eliciting such a response involves presentation of the antigen at the mucosal surface. In general parenteral vaccines (Newby \& Stokes, 1984b) have not been effective, and where exceptions exist they almost always involve some mucosal component (e.g. oral + parenteral). The other exception to this principle involves immunization of the dam to achieve passive protection of the sucking neonate, by virtue of the presence of antibodies in colostrum. In the case of domestic species, antibodies in colostrum are derived from serum and these may be effectively stimulated by parenteral immunization. Unlike colostrum, milk antibodies are locally produced and in this case, like other true mucosal secretions, mucosal immunization is required to stimulate production of antibodies (Newby \& Stokes, 1984b).

Having established that mucosal vaccines are those of choice for both theoretical and practical reasons, it is, however, disappointing that it is surprisingly difficult to stimulate a vigorous, protective response via this route. As has already been pointed out, this is of 
benefit with regard to the 'harmless antigens' (e.g. dietary antigens) also presented to the mucosae. To date the majority of effective mucosal vaccines are living attenuated organisms reflecting the now generally held axiom that either replication or at least adherence are essential prerequisites of mucosal immunogenicity. Whilst there is now clear evidence of memory in the secretory immune system, vital if any vaccination regimen is to be effective, it has also been shown that the response is highly dose-dependent. Thus where dead oral vaccines have been used, very large numbers of bacteria are required and even then the level of immunity is sub-optimal by comparison with that following the use of live organisms (Newby \& Stokes, 1984b). Another problem associated with mucosal immunization, unique to the gastrointestinal tract, relates to an ability to pass through the stomach in a form that remains antigenic. In this context the use of capsules or liposomes (Gregoriadis, 1981) may be of benefit. Alternatively the observed immunological link between mucosal sites, such that oral immunization leads to the appearance of antibodies in milk, may be of benefit.

The new vaccines. Vaccines derived as products of biotechnology may take a variety of forms and rather than discussing them on a specific disease basis it is proposed to briefly describe the general feature of each type.

The genes coding for protein or peptide antigens derived from a number of bacteria and viruses have been cloned and expressed in appropriate bacteria. For example, with the foot and mouth disease virus one of the structural capsid proteins, VPI, has been identified as carrying the antigenic determinants necessary to raise neutralizing antibodies in experimental animals (Kupper, 1984). The viral RNA encoding for VPI has been copied into double-stranded cDNA, inserted in a bacterial vector and expressed in E. coli (Kupper et al. 1981). Subsequently a range of oligopeptides from VPI have been isolated or synthesized and a major antigenic determinant comprising nineteen amino acids identified. The question that remains is how these proteins or peptides may be presented to the immune system in order to stimulate a protective response. The isolated VPI protein, for instance, is not as antigenic as the virus and a 100 to 1000-fold excess on a molar basis is required in order to obtain satisfactory protection. With the oligopeptide it is necessary to couple to a carrier protein (e.g., KLH) and although a single injection of $200 \mu \mathrm{g}$ has been shown to protect guinea-pigs from virus challenge, to date no satisfactory method has been found to stimulate a protective response in cattle. Similar results have been obtained with $E$. coli antigens (K88ab, ac, ad and $\mathrm{K} 99$ have all been cloned and expressed (Storm, 1984)) but it still remains to be determined how a protective mucosal response may be stimulated. Whilst to date this synthetic peptide vaccine approach has been disappointing, its theoretical attraction over conventional dead vaccines remains since it avoids the requirement for handling large volumes of infectious materials, or vaccinal strains that grow poorly in bulk-suspension cultures, and the risk associated with failure to completely inactivate the virus before incorporation into a vaccine.

An alternative approach involves the use of attenuated strains and these may take a variety of forms, and have been used against a number of agents. Deletion mutants of Aujeszky's disease virus have been prepared (van Oirschot et al. 1985) and although shown to have greatly reduced virulence are still highly immunogenic in pigs. Similarly an attenuated strain of Salmonella typhi, Ty21a, which is unable to utilize galactose, has been derived (Germanier \& Furer, 1975). The organism is able to survive for a limited number of divisions, but antigenicity remains unaltered. For another enteric pathogen, cholera, an appropriate mutant is also available (Honda \& Finkelstein, 1979). In this case it produces only the B subunit of the toxin and thus, whilst being able to bind to the epithelial cell and give good protection, does not generally produce diarrhoea. 
Where attenuated strains of a particular organism are not available or appropriate the use of live recombinant vaccine may be of benefit. Intranasal inoculation with recombinant Vaccinia virus has been shown to protect mice against upper and lower respiratory tract infections with influenza (Small et al. 1985). Alternative vectors to Vaccinia are also available, for example yeasts or engineered non-pathogenic strains of $E$. coli that produce only the B subunit of the labile toxin, and so bind to enterocytes but do not produce diarrhoea.

The general acceptability of live attenuated vaccines remains to be determined and the possibility of developing techniques whereby peptide vaccines can be delivered in a way that stimulates a protective mucosal response remains attractive. In this context the use of peptide conjugates coupled to molecules such as cholera toxin or lectins that bind avidly to epithelial cells may be of benefit. Alternatively the potential role of antiidiotypic antibodies (monoclonal?) may be of interest, it having been recently shown that such antibodies may prime for protection against E. coli (Stein \& Soderstrom, 1984).

The capacity of biotechnology to produce a range of antigenic moieties derived from infectious agents would appear to be unlimited. Further, it should not be difficult to identify those antigens that are important in the pathogenesis of disease. The major problem that remains is how best these products might be presented to the immune system in order to stimulate a protective immune response. It is therefore to this target of understanding how antigens are presented to and processed by the mucosal immune system that intense future research should be directed.

The financial support of the AFRC is gratefully acknowledged.

\section{REFERENCES}

Germanier, R. \& Furer, E. (1975). Journal of Infectious Diseases 131, 553-564.

Gregoriadis, G. (1981). Lancet ii, 241-247.

Honda, T. \& Finkelstein, R. A. (1979). Proceedings of the National Academy of Sciences, USA 76, 2052-2059.

Kupper, H. A. (1984). In New Technology for Animal Health and Production, AVI Symposium, pp. 43-57

[D. B. Ross and K. C. Sellers, editors]. Rochester: Staples Printers Rochester Ltd.

Kupper, H. A., Keller, W., Kurz, Ch., Forss, S., Schaller, H., Franze, R., Strohmaier, K., Marquardt, O., Zaslausky, V. G. \& Hofocheirder, H. (1981). Nature 289, 555-559.

Miller, B. G., Newby, T. J., Stokes, C. R. \& Bourne, F. J. (1984). Research in Veterinary Science 36, 187-193. Muscoplat, C. C. (1983). Bovine Proceedings no. 15, 8-14.

Newby, T. J. \& Stokes, C. R. (1984a). Local Immune Responses of the Gut. Boca Raton, USA: CRC Press.

Newby, T. J. \& Stokes, C. R. (1984b). Veterinary Immunology and Immunopathology 6, 67-105.

Secher, D. S. \& Burke, D. C. (1980). Nature 285, 446-450.

Small, P. A., Smith, G. L. \& Moss, B. (1985). In Vaccine 85, pp. 175-176 [R. A. Lerner, R. M. Chanock and F. Brown, editors]. New York: Cold Spring Harbor.

Stein, K. E. \& Soderstrom, T. (1984). Journal of Experimental Medicine 160, 1001-1011.

Stokes, C. R., Miller, B. G. \& Bourne, F. J. (1986). In Food Allergy and Intolerance, pp. 286-300 [J. Brostoff and S. J. Challacombe, editors]. London: Bailliere Tindall.

Storm, P. K. (1984). In New Technology for Animal Health and Production, AVI Symposium, pp, 37-42 [D. B. Ross and K. C. Sellers, editors]. Rochester: Staples Printers Rochester Ltd.

Taylor, G., Stott, E. J., Bew, M., Fernie, B. F. \& Cote, P. J. (1983). Lancet ii, 976.

van Oirschot, J. T., Quint, W. G. V., Gielkens, P. W., de Leeuw, P. W. \& Berns, A. J. W. (1985). In Immunity to Herpes Virus Infections of Domestic Animals, pp. 281-290 [P. P. Pastoret, E. Thiry and J. Saliki, editors]. Luxembourg: CEC. 